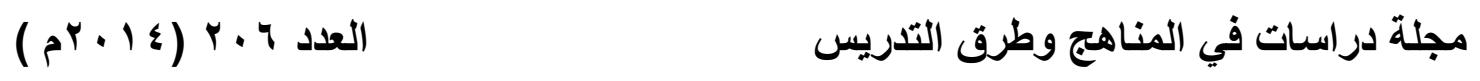

\title{
Einsatz der Mindmap und des kooperativen Schreibens im Vergleich zur Entwicklung der Schreibkompetenz bei den Lernenden der Deutschabteilung an der Pädagogischen Fakultät
}

Duaa Abd El Karim abd El Regal Ahmed

Assistent Professorin an der Curricula und Methodikabteilung (DaF)

Pädagogische Fakultiät - Helwan Universität 


\section{Einsatz der Mindmap und des kooperativen Schreibens im Vergleich zur Entwicklung der Schreibkompetenz bei den Lernenden der Deutschabteilung an der Pädagogischen Fakultät \\ - Ass. Prof. Dr. Duaa Abd El Karim}

\section{Abstract}

Schreiben als produktive Fertigkeit beim Lernen einer Fremdsprache erfordert mehr Anwendung und Übung. Die vorliegende Arbeit hat zum Ziel, Schreibschwierigkeiten beim Verfassen von Texten zu bewältigen, und die Schreibkompetenz bei den Lernenden der Deutschabteilung an der Pädagogischen Fakultät zu entwickeln. Die Arbeit beschäftigt sich lediglich mit zwei Schreibverfahren (der Mindmap und dem kooperativen Schreiben), um ihre Auswirkung auf das Erlernen von Schreiben zu erkennen, und zu vergleichen. Am Beispiel von Mindmap oder von kooperativem Schreiben fokussiert die Arbeit die folgenden Schreibprozesse; Planen - Vorbereiten - Organisieren Formulieren und Überprüfen. Die Arbeit beschränkt sich auf drei Textsorten: Aufsätze, offizielle Briefe und persönliche Briefe.

Die Kandidatin hat einen Schreibtest entworfen, um die Schreibkompetenz zu bewerten. Der Test gilt als Vor- und Nachtest und bewertet das Niveau der zwei Testgruppen vor den Modulen. Das Ergebnis des Vortests ist, dass es keinen Unterschied zwischen dem Niveau der zwei Testgruppen vor der Umsetzung der Module gibt. Danach beginnt die Kandidatin, die Module zu unterrichten. Die Analyse der Ergebnisse untersucht und vergleicht die Wirksamkeit der zwei Verfahren. Kooperatives Schreiben hat eine größere Wirkung als Mindmap auf die Entwicklung von Schreibkompetenz. Eine quantitative und qualitative Analyse der Ergebnisse findet in der Arbeit statt.

Schlagwörter: Mindmap - Kooperatives Schreiben - Textproduktion Schreibkompetenz 


\section{Einleitung und Problemstellung}

Das Lernen einer Fremdsprache bedeutet, dass sprachliche Kompetenzen beherrscht werden. Das Lernen von Deutsch als Fremdsprache lässt die Lernenden in der Lage sein, Kenntnisse und Erfahrungen über Hören, Lesen, Sprechen und Schreiben zu erwerben. Im DaF-Unterricht haben die sprachlichen Fertigkeiten die gleiche Gewichtung. Wenn sie die gleiche Gewichtung nicht haben, bleibt das Ziel vom Lernen einer Fremdsprache, dass die Lernenden die Sprache gebrauchen können und sich selbst in der Fremdsprache äußern können.

Im folgenden Beitrag wird die Entwicklung der Fertigkeit "Schreiben" beim Erwerb von Deutsch als Fremdsprache fokussiert. Schreiben, als komplexer Produktionsprozess ist eine wichtige Kompetenz, die deshalb einen wichtigen Platz im FSU hat. Weiterhin hat Schreiben auch eine kommunikative Funktion. Im Schreiben haben die Lernenden die Möglichkeit, Ausdrücke und Texte herzustellen. Mit anderen Worten äußern sie ihre Gedanken, Meinungen und Einstellungen. Sie führen eine schriftliche Kommunikation. Sie können folglich mit Kreativität und Fantasie schreiben.

Um den Erwerb der Schreibkompetenz zu erreichen, sollten die Lernenden wissen, wie sie schreiben, was sie schreiben und warum sie schreiben. Deshalb ist der Einsatz bestimmter Schreibverfahren bzw. Schreibtechniken im Unterricht gefragt. Die Lernenden sollen das Schreiben und das Produzieren von Texten üben. Deshalb wählt der Lehrer effektive interessante Lehr- und Lernstrategien und Lehr- Lerntechniken, um die Schreibkompetenz zu entwickeln. Um gute Erfolge zu erzielen, müssen die Lernenden gut motiviert sein und dürfen nicht unterfordert 
werden. Die folgende Arbeit untersucht und vergleicht zwei Schreibstrategien; Mindmap und Kooperatives Schreiben, die als interessante Verfahren betrachtet werden und das Interesse für das Schreiben erhöhen können.

\subsection{Problemstellung der Arbeit}

Das Studium an der Pädagogischen Fakultät zielt darauf, eine kompetente Lehrerausbildung anzubieten und gute Lehrer auszubilden. Beim Studium werden die sprachlichen Kompetenzen entwickelt. Die Lernenden finden, dass einige sprachliche Kompetenzen schwer zu erlernen bzw. zu beherrschen sind wie z. B. Sprechen und Schreiben, weil sich bei den Lernprozessen der beiden Fertigkeiten Komplikationen ergeben. Die Lernenden bemühen sich, Ausdrücke und Texte auf Deutsch zu produzieren.

Die Ergebnisse einer aus Unterrichtsbeobachtungen und offenen Interviews mit den Lernenden an der Deutschabteilung bestehenden Pilotstudie weisen darauf hin, dass ein hoher Bedarf am Erwerb Fertigkeit Schreiben besteht. Die Lernenden wollen in der Lage sein, mit Deutsch schriftlich zu kommunizieren. Sie haben Interesse daran, mehr über Schreiben und Schreibphasen zu lernen. Oft haben die Lernenden aber Probleme mit dem Schreiben; sie beherrschen den richtigen Schreibstil nicht, sie haben Schwierigkeiten die passenden Wörter zu finden, manchmal haben sie keine Ideen, was sie schreiben sollen.

\subsection{Problemstellung}

Schreiben als komplexer Produktionsprozess im FSU wird trotz der betonten Bedeutung der Entwicklung von sprachlichen Kenntnissen vernachlässigt. Es fehlt den Lernenden an Wissen $\mathrm{zu}$ logischen 
systematischen Schreibphasen bzw. -schritten. Das führt dazu, dass die Lernenden Hindernisse haben, deutsche Texte schriftlich zu produzieren, und ihre Gedanken, Meinungen und Einstellung erfolgreich auszudrücken.

\subsection{Fragen der Arbeit}

Um dieses Problem zu behandeln und zu lösen, versucht die Arbeit, Antworten auf die folgenden Fragen zu finden:

1. Welche Schreibschwierigkeiten begegnen den Lernenden an der Deutschabteilung?

2. Wie sehen ein Modul im Licht der Mindmap und ein Modul im Licht des Kooperativen Schreibens aus, um das Schreiben zu entwickeln?

3. In wieweit wirken sich die zwei Schreibstrategien (Mindmap Kooperatives Schreiben) auf die Entwicklung der Schreibkompetenz bei den Lernenden des zweiten Studienjahrs an der Deutschabteilung aus?

\subsection{Eingrenzung der Arbeit}

Die vorliegende Arbeit beschränkt sich auf;

- Die Lernenden der Deutschabteilungen im zweiten Studienjahr an der Pädagogischen Fakultät der Helwan Universität, weil sie die Grundlagen besitzen, um unterschiedliche Texte zu schreiben. Das Niveau der Lernenden ist von B1 bis B2.

- Zwei Schreibstrategien (Mindmap - Kooperatives Schreiben) als Lösungen der Schreibprobleme. Die Auswahl von solchen Schreibverfahren hängt von den Schreibschwierigkeiten $a b$, die die Lernenden haben.

\subsection{Zielsetzung der Arbeit}

Die Arbeit setzt sich folgende Ziele: 
- Einige Schreibschwierigkeiten zu behandeln und Lösungen zu finden.

- zur Verbesserung der sprachlichen Fertigkeit "Schreiben" beizutragen.

- die Wirkung der zwei Schreibstrategien zu untersuchen und zu vergleichen.

\subsection{Hypothesen der Arbeit}

- Es sind abweichende Ergebnisse zwischen den Mittelwerten bzw. den Rängen der ersten Testgruppe (Mindmap) im Vor- und Nachtest bezüglich der Schreibkompetenz zugunsten des Nachtests zu erwarten.

- Es sind abweichende Ergebnisse zwischen den Mittelwerten bzw. den Rängen der zweiten Testgruppe (kooperatives Schreiben) im Vor- und Nachtest bezüglich der Schreibkompetenz zugunsten des Nachtests zu erwarten.

- Es sind abweichende Ergebnisse zwischen den Mittelwerten bzw. Rängen der ersten Testgruppe (Mindmap) und der zweiten Testgruppe (Kooperatives Schreiben) im Vor- und Nachtest bezüglich der Schreibphasen (Planen - Vorbereiten - Organisieren - Strukturieren Überdenken) zugunsten des Nachtests zu erwarten.

\subsection{Terminologie}

\subsubsection{Schreiben}

Schreiben wird als Prozess definiert und verstanden, der neben der Planung und Formulierung eines Textes auch die Überarbeitung binhaltet. Schreiben, das auch als komplexe Handlung bezeichnet wird, besteht aus aufbauenden Schritten, die miteinander verbunden sind. Durch diese Schritt können sich die Lernenden ausdrücken, Gedanken ordnen und strukturieren. Der Schreibprozess besteht aus den folgenden Subprozessen (Planen - Formulieren - Überarbeiten) (Benó 2011: 83). 
Kaishauri (2014: 7-8) fasst die Schritte im Schreibprozess wie folgt zusammen: Schritt 1: Ideen sammeln und gruppieren, Schritt 2: Ideen aussortieren und strukturieren, Schritt 3: Die Reihenfolgen der Argumente festlegen, Schritt 4: Jeden Gedanken in einem Satz ausformulieren, Schritt 5: Die Sätze ausbauen und miteinander verknüpfen, Schritt 6: Den Text lesen und (falls notwendig) redigieren bzw. ergänzen.

\subsubsection{Mindmap}

Neuhaus (2001: 22) betrachtet Mindmap als Form der Textplanung. Neumann (2014: 5) definiert Mindmap als eine baumartige grafische Darstellung, die die Beziehungen zwischen verschiedenen Begriffen aufzeigt. Die Mindmap bezeichnet man als eine Gedächtnislandkarte, sie verbindet sich mit Lerntheorien, sie ist eine Strategie zur Sammlung und Organisation von zusammenhängenden Gedanken, die zur Text-produktion führen (Heuer 2002: 2; Reich, K 2003: 2).

\subsubsection{Kooperatives Schreiben}

Das kooperative Schreiben wird als eine Strategie des Schreibens (Schreibstrategie in der Schule) betrachtet (Neuhaus 2001: 8). Einige Forschungen bearbeiten den Begriff "kooperatives Schreiben" und bieten ihn als Sozialform im Unterricht an (ebd: 9). Unter kooperativem Schreiben versteht man, dass die Lernenden gemeinsam im Team eine Aufgabe erledigen können, es betrachtet auch als Strategie des gemeinsamen Lernens (Kruse 2010: 161).

\subsection{Methodisches Vorgehen}

Die vorliegende Arbeit verfolgt systematische Schritte, um das Ziel zu erreichen. Sie sind: 
- Sichtung der Literaturen in den folgenden Bereichen: Deutsch als Fremdsprache, das Erwerben von sprachlichen Fertigkeiten, Schreiben, Schreibverfahren Mindmap und Kooperatives Schreiben.

- Bestimmung der Schreibschwierigkeiten von Lernenden an den Deutschabteilungen durch einen Fragebogen.

- Bestimmung der Schreibphasen, die die Lernenden in jedem Modul erwerben und ausüben sollen.

- Erstellung zweier vorgeschlagener Lernmodule im Lichte der Schreibstrategien (Mindmap - Kooperatives Schreiben) zur Förderung von Schreiben. Einige Schritte werden verfolgt: Ziele bestimmen, Texte und Schreibthemen auswählen, Lehr-Lernmethoden bestimmen, Aktivitäten und Aufgaben erstellen und entwickeln, Verfahren zur Evaluation auswählen.

- Um den Effekt der zwei Schreibstrategien festzustellen, wird das experimentelle Design (zwei Testgruppe) ausgewählt.

Erstellung eines Schreibtests, Umsetzung des Tests vor den Modulen, Umsetzung der zwei Module, Umsetzung des Tests nach den zwei Modulen.

- Statistische Auswertung der Ergebnisse, Schlussfolgerungen und Konsequenzen für Schreiben und Sprachenlernen.

\section{Theoretischer Teil}

\subsection{Schreiben im Fremdsprachenunterricht}

Das Schreiben im DaF- Unterricht kann einen großen Beitrag zur sprachlichen Kommunikation leisten. Das Schreiben als komplexer Prozess verfolgt Schritte, präsentiert orientierte Handlung und fordert kooperative Arbeitsweisen (Neuhaus 2001: 10). Darüber hinaus denken die Lernenden 
im Schreibprozess über Themen nach, über die sie schreiben wollen, und sie bemühen sich, das Thema und ihre Gedanken genau darzustellen (Kruse 2010: 56). Im Schreibprozess bieten sich den Lernenden viele Vorteile. Ciepielewska- Kaczmarak (2011: 276-277) fasst einige Vorteile vom Schreiben im DaF- Unterricht zusammen. Diese Vorteile sind die Wirkung des Schreibens auf die Motivation der Lernenden und auf ihre Lernfortschritte.

Das Schreiben steht im Zusammenhang mit den anderen Fertigkeiten. Die Lernenden können mit Hilfe von sprachlichen Aktivitäten wie Texte zuhören, Geschichte erzählen, aus Büchern vorlesen das Schreiben gut lernen (ebd: 280). Die Lernenden sollen auch das Schreiben nicht als eine einfache Aufgabe betrachten, sondern als eine Aufgabe, die vielfältige Funktionen erfüllt (Hinrichs 2011: 3). Deshalb erfordert das Schreiben als eine produktive komplexe Fertigkeit bestimmte Qualifikationen vom Schreiber. Beim Schreiben können verständliche Texte erstellt werden, die ein Weg zur Kommunikation mit anderen sind. Die Situationen, in denen die Lernenden das Schreiben praktizieren, befördern auch Positivität, Flexibilität und Lust am Schreiben (Böttcher 2013: 2).

Schreiben bezeichnet man als strategische Kompetenz, weil der Schreibprozess durch aufeinander und miteinander verbundenen Phasen strukturiert ist. Diese Phasen charakterisieren den Schreibprozess. Sie sind: Fokussieren, Generieren, Anpassen, Strukturieren, Formulieren und Überdenken (Reinhold 2011: 189). Die Phasen des Schreibprozesses nennt man auch Schreibschritte. Die Phasen geben den Lernenden die Möglichkeit, die Gedanken zu ordnen und zu strukturieren, über ihre Erfahrungen zu berichten und ihre Meinungen zu äußern. Die Subprozesse 
Planen, Formulieren und Überarbeiten werden ebenfalls beim Schreiben hervorgehoben (vgl. Benó 2011: 83). Im Schreibprozess bearbeiten die Lernenden Texte. Bei der Formulierung eines Textes vertiefen die Lernenden ihre Sprachkenntnisse und Erfahrungen. Damit die Lernenden den Umgang mit unterschiedlichen Texten lernen, sollte der Lehrer verschiedene Textsorten im Unterricht behandeln. Jeder Text hat einen bestimmten Zweck und eigene Eigenschaften (Trim et al. 2001: 95). Die Verschiedenheit von Textsorten eröffnt die Möglichkeit, Schreibkompetenz zu fördern. Die Auswahl einiger Textsorten wird nach Kriterien "wie z. B. Aktualität, Niveau der Lernenden und Relevanz für die Lebenswelt der Lernenden" festgestellt.

Alle Texte, die von Lernenden produziert werden, sollen bewertet werden. Es gibt eine Menge von Bewertungskriterien, um die produzierten Texten der Lernenden einzuschätzen. Der Schreiber kann seine Leistung im Schreiben selbst durch diese Bewertungskriterien reflektieren. Es ist immer möglich, eine Selbst- oder Fremdbewertung vorzunehmen; Beispiele dieser Kriterien sind: Gestaltung, Qualität, Inhalt ..... usw. (Steffens 2001: 37). Der Schreibprozess geschieht Schritt für Schritt durch Anwenden und Üben. Deshalb erwartet der Lernende in jeder Phase eine Bewertung und Feedback. Durch Hinweise, Bemerkungen und Rückmeldungen kann der Schreibprozess verbessert werden.

Im Schreibprozess sollen neue geeignete und unterschiedliche Methoden angewandt werden. Anhand dieser Methoden bzw. Schreibverfahren werden die Ausdrücksmöglichkeiten der Lernenden entwickelt (Canakkale 2012: 155). Schreibverfahren wie Mindmap können eine aktivierende Wirkung haben; es regt Interesse und Fantasie der Lernenden an, 
Kooperatives Schreiben erleichtert soziales Lernen und fördert Selbstvertrauen. Beide Verfahren können einen Beitrag zum Schreiberwerb leisten.

\subsection{Die Mindmap zur Textproduktion}

Es ist sinnvoll, die Gedanken im Zusammenhang zu erstellen. Das führt dazu, dass die Lernenden die gelernten Inhalte leichter wiederholen bzw. wiedergeben können. Assoziation der Gedanken durch Mindmap hilft den Lernenden dabei, dass sie von Wörtern zu Sätzen finden (Heuer 2003: 2). Die Mindmap unterstützt die Lernenden bei der Strukturierung von Ideen, die auch durch Bilder, Symbole und Zeichnungen visualisieret werden können (www.post.ch/direkt/point20,1,2014.S,2). Zusätzliche Visualisierung wie Farbe macht die Darstellung von Gedanken interessant. Durch Verwendung von Symbolen und Zeichnungen bei der Erstellung einer Mindmap werden die Informationen, Gedanken und Inhalte schnell und nachhaltig im Gehirn gespeichert. Die Formulierung von Gedanken führt danach zur Organisation und dann zur Strukturierung von Texten.

Wegen der variationsreichen Erweiterungen einer Mindmap unterscheidet sie sich vom Wortigel. Beim Wortigel findet man lediglich Wörter. Mit Hilfe einer Mindmap sammelt man erweiterbare Ideen zum zusätzlichen Begriff. Dabei ist es möglich, unwichtige Ideen zu reduzieren. Mindmap unterscheidet auch vom (Cluster). Mit einem Cluster werden alle Wörter, Gedanken, Ideen, Zitaten, Gefühle, Informationen mit Pfeilen, Linien und Kreisen untereinander verbunden, und werden in Beziehung mit dem Ausgangsbegriff und auch in Beziehungen mit einander gesetzt. Es ist auch möglich, ein Wort mit einem andern zu verbinden und zu vernetzen (Bürkl et al. 2013: 31).

\section{$\bullet \bullet$}


Mindmaps können unterschiedliche Funktionen im Fremdsprachenunterricht erfüllen. Neumann (2012: 5) berichtet von der Wichtigkeit beim Einsatz von Mindmaps beim Lernen, auf die der Forscher Tony Buzan hingewiesen hat. Dazu zählt z. B., die Leistungsfähigkeiten des Gehirns erhöht werden, und der Lernerfolg realisiert werden kann. Ein anderes Beispiel für die Funktion von Mindmaps ist die Ideenanalyse und Ideenbearbeitung, die aus gelesenen Texten hergestellten werden können. Mindmaps beim Schreiben werden aber verwendet, um Gedanken und Texte zu produzieren. Die Mindmap gilt als ein Weg zu Kreativität, kognitiv anspruchsvoller Arbeit, Freude und Fantasie im Unterricht.

Texte zu bearbeiten und Texte zu gestalten im Unterricht sind Ziele des Fremdsprachenlernens. Die passenden Techniken zur Textbearbeitung oder Textgestaltung sollte der Lehrer festlegen. Er sollte Methoden und Techniken entwerfen, die das Lernen der Fremdsprache und die Sprachfertigkeiten erleichtern, die Freiheit des Lernenden in der Praxis ermöglichen und insbesondere das Schreiben fördern (Canakkale 2012: 155). Mindmap bezeichnet man als eine dieser Methoden. Deshalb sind alle logischen Formen zum Lernen und zum Erstellen von Mindmaps erlaubt. Durch bestimmte Schritte wird eine Mindmap gestaltet. Diese Schritte sind: Schritt 1: Das Thema, die Idee oder den Kernbegriff in die Mitte schreiben. Schritt 2: Linien ziehen, ein Wort auf jede Linie schreiben. Die Linien für Vorteile, Nachteile, Merkmale, Gründe usw. ziehen.

Schritt 3: Oberbegriffen festlegen, dann Fragen und Lösungen formulieren. Schritt 4: Gedanken ordnen, das Unwichtige reduzieren und die Gedanken durch Symbole und Zeichnungen erweiteren. 
(www.post.ch/direkt/point20.1,2014,S,3;

www.bog.admin.chttranspk.15.1.2014,S,1).

Eine Mindmap soll gewisse Kriterien oder Prinzipien verfolgen. Die Kriterien sind z. B. Qualität, Fixierung, Farbe, Schrift, Bilder, Medium (Reich 2003: 4). Trotzdem gibt es keine einschränkenden Darstellungskriterien. Jeder kann für persönliche Ziele Mindmap darstellen. Der Lehrer kann aber die Mindmap der Lernenden bewerten und die beste auswählen. Dieser Wettkampf motiviert zum Lernen und fördert die Bereitschaft zur Gestaltung richtiger Mindmaps mit Sorgfalt. Einige Bewertungskriterien betont Thiel (2014: 7) wie z. B. Lesbarkeit, Übersichtlichkeit, Klarheit, angemessene Informationsdichte und richtige Schreibweise. Es ist auch wichtig, sich nicht nur auf die Form der Mindmap sondern sich auch auf die anhand der Mindmap fertig produzierten Texte zu konzentrieren.

\section{3. kooperatives Schreiben vs. allein schreiben}

Kooperatives Schreiben im DaF-Unterricht als Schreibstrategie wirkt sich auf die Motivation der Lernenden aus. Die Mitglieder einer Gruppe schlagen unterschiedliche Ideen vor, diskutieren miteinander, und tauschen ihre Sprachkenntnisse und eigne Erfahrungen aus (Benó 2011: 79). Kooperatives Schreiben fördert Teamfähigkeit und eingemessenes Sozialverhalten. Heutzutage stehen ein positives Unterrichtsklima und Kooperation beim Lernen einer Fremdsprache im Vordergrund, wobei letzteres den Lernenden mehr Selbstvertrauen verschafft.

Wenn der Lehrer das Schreiben im DaF- Unterricht entwickeln möchte, sollte er sich neue didaktische innovative Ansätze überlegen, die das Verfassen eines Textes erleichtern können, die Lernenden mit Freude und ohne Angst schreiben lassen, und die helfen, dass sich Lernende gut und 
kreativ ausdrücken können (Canakkale 2012: 155). Beim kooperativen Schrieben hat jedes Mitglied eine Aufgabe oder eine Rolle. Der Lehrer spielt eine Rolle, aber nicht traditionell. Der Lehrer organisiert den Schreibprozess, gibt auch Schreibimpulse, erfindet eine gute Schreibatmosphäre und bewertet das Schreiben der Lernenden (Steffens 2001: 73). Die Lernenden übernehmen die Verantwortung für ihr Lernen und das Textprodukt, das sie produzieren (Sturm 2008: 4). Sie haben die Hauptrolle in den verschiedenen Schreibprozessphasen. Diese sind im Folgenden zusammengefasst: a) Schreibmotivation, b) Themenfindung, c) Schreibaufgabe und Schreibproblemerklärung; logisch - semantische Klärung, d) Linearisierungsplan; Textmuster - wie man einen Text schreibt, e) Textausführungen; Bausteine - Versionen - Anfänge und Schlüsse - Vorschieben, f) Überarbeitung (Schwarzbauer et al. 2010: 11).

Es gibt zwei Arten des kooperativen Schreibens: Das gemeinsame kooperative Schreiben und das schrittweise kooperative Schreiben. Beim gemeinsamen kooperativen Schreiben übernehmen die Lernenden die Verantwortung gemeinsam für den Text. Beim schrittweisen kooperativen Schreiben schreibt einer der Lernenden einen Text und dann integriert mit anderen Personen, um das Geschriebene $\mathrm{zu}$ kommentieren und $\mathrm{zu}$ bewerten. Diese Fremdbewertung hilft bei der Überarbeitung des eigenen Textes (Gavrilas 2012: 7-8).

Um das kooperative Schreiben im DaF- Unterricht durchzuführen, bietet der Lehrer den Lernenden einige Schritte an. All diese Schritte garantieren die gemeinsame Problemlösung bei der Bewältigung von Schreibaufgaben. Der Lehrer könnte sequenzielles Schreiben als erste Aufgabe vorgeben. Das heißt; dass ein Mitglied nach dem anderen an der Aufgabe arbeitet. 
Paralleles Schreiben heißt jedoch, dass jedes Mitglied eine bestimmte Teilaufgabe hat, was mehr Organisation und Koordination verlangt (Sturm 2008: 1-2). Es ist auch wichtig zu betonen, dass die Merkmale des kooperativen Schreibens im Laufe der Arbeit festgelegt werden. Am Ende des kooperativen Schreibens stehen immer Schreibkonferenzen, worin die Lernenden das Geschriebene und den produzierten Text miteinander bearbeiten und bewerten, Diskussionen über den Text führen, und Kommentare geben. Die Mitlernenden finden in den Schreibkonferenzen Hinweise und Vorschläge für ihre eigenen Texte (Steffens 2001: 69). Der Lehrer kann irgend eine Form vom kooperativen Schreiben einsetzen, weil die Lernenden gleichermaßen und bereit sind, gemeinsam zu schreiben.

\section{Forschungsinstrumente}

In diesem Teil geht es um die praktische Durchführung zur Entwicklung von Schreibkompetenz. Zunächste werde ich die Schreibschwierigkeiten bestimmen. Ich habe die Frage gestellt, welche Schreibschwierigkeiten den Lernenden im Laufe des Studiums begegnen. Es ist wichtig, die Schreibschwierigkeiten zu erkennen. Anhand dieser Schwierigkeiten sucht man passende Lösungen für Schreibschwierigkeiten und Schreibblockaden. Aus diesem Grund habe ich die zwei Schreibverfahren (Mindmap und kooperatives Schreiben) untersucht. Im Folgenden werden zwei Lernmodule anhand dieser zwei Verfahren aufgebaut. Danach wird einer Schreibtest entwickelt, um die Schreibkompetenz zu bewerten.

\subsection{Bestimmung von Schreibschwierigkeiten}

Ich habe einen Fragebogen zur Bestimmung von Schreibschwierigkeiten bei Lernenden anhand der von Wolfrum (2010: 77) festgelegten Schreibschwierigkeiten entwickelt. Durch diesen Fragebogen habe ich die 
häufigsten Schreibschwierigkeiten bei den Lernenden an der Deutschabteilung in zweiten, dritten und vierten Studienjahren angemacht können. (S. Anhang 1)

\subsubsection{Die Ergebnisse nach der Umsetzung des Fragebogens}

Die Umsetzung des Fragbogens zeigt die wichtigsten Schreibschwierigkeiten beim Schreibprozess und beim Produzieren von Texten. Die häufigsten Schreibschwierigkeiten bei den Lernenden werden im Folgenden erörtert:

- Schwierigkeiten im Umgang mit bestimmten Textsorten / einem bestimmten Schreibstil.

- Übertragung aus der Muttersprache, Schwierigkeiten mit Wortschatz und Formulierungen.

- Probleme bei der Verschriftlichung von Gedanken (falscher Gebrauch von Metaphern).

- Mit dem Schreiben beginnen: Fehlende Schreibideen.

- Textbausteine: Allgemeingültige, unpassende Formulierungen.

Anhand von diesen Befunden habe ich zwei Strategien zum Schreiben praktiziert, die Auswahl der Schreibstrategien hängt von Schreibschwierigkeiten $\mathrm{ab}$, die von Wolfrum 2010 bestimmt werden, um solche Schwierigkeiten zu bewältigen.

\subsection{Aufbau von Modulen}

Das Ziel der zwei Module liegt darin, dass die Lernenden Motivation, Lust, Interesse am Schreiben bekommen. Das erleichtert die Entwicklung von Schreibkompetenz. Am Anfang wird die Schreibkompetenz bestimmt. Die Schreibkompetenz wird in den Lernmodulen in Schreibphasen

\section{7.}


unterteilt. Sie sind: Planen - Vorbereiten - Organisieren - Formulieren Überlegen. (S. Anhang 2)

\subsubsection{Phasen der Lernmodule}

Die beiden Lernmodule sind in ihrem Aufbau ähnlich. Jedes Modul besteht aus fünf Phasen. Jede Phase besteht aus dem Folgenden: Lernzeile, didaktisches Vorgehen und Aufgaben im Unterricht. Die beiden Lernmodule bearbeiten die selben Textsorten: offizielle Briefe, persönliche Briefe und Aufsätze. Die beiden Lernmodule haben die gleichen Aufgaben, wobei sie aber von einander verschieden sind. Sie verfolgen unterschiedliche Schritte, da sie vom Schreibverfahren abhängen. (S. Anhang 3), (S. Anhang 4)

\subsubsection{Die Umsetzung der Module}

Es gibt zwei Gruppen von Lernenden. Eine Gruppe arbeitet mit Mindmap, die andere Gruppe arbeitet kooperativ mit kooperativem Schreiben. Jede Gruppe setzt sich aus 19 Lernenden zusammen. Ich unterrichte die zwei Lernmodule jew. vier Stunden für jede Gruppe pro Woche seit Beginn des zweiten Semesters im Studienjahr 2013 / 2014, das 6 Wochen dauert.

\subsection{Schreibtest}

Die Formulierung von Testaufgaben repräsentiert die fünf Phasen des Schreibens und die Lernziele der Lernmodule. Der Test wird vor und nach den Lernmodulen umgesetzt. Der Test hat das Ziel, die Schreibkompetenz zu bewerten. Der Test besteht aus 5 Aufgaben. Jede Aufgabe betrifft eine Schreibphase bzw. einen Schreibschritt.

Der Wert des Korrelationskoeffizienten darf nicht mehr als 1.00 sein. Es ergibt sich, dass die Zuverlässigkeit des Tests anhand vom "Wilcoxon 
Koeffizient" 0.65 ist. Diese Ergebnisse zeigen, dass der Test valide ist. (S.

\section{Anhang 5)}

\subsection{Musterantwort und Bewertungskriterien zum Schreiben}

Die erste Aufgabe ist eine geschlossene Frage, die nur eine richtige Antwort hat. Die Lernenden wählen das passende Wort. Die zweite Frage bewertet die Vorbereitung von Gedanken, d. h; wie die Lernenden Gedanken zu einem Thema formulieren können. Die Kriterien, die ich verwendet habe, um Gedanken zu bewerten, sind:

1. Die Zahl der Gedanken, 2. Das Variieren der Gedanken, 3. Richtigkeit und Klarheit der Gedanken, 4. Abhängigkeit vom und Zusammenhang mit dem Thema. Die Aufgabe 3 bewertet das Ordnen und das Organisieren von Gedanken. Die Frage ist eine geschlossene Frage.

Die Aufgabe 4 verlangt hinein Kriterienkatalog für die Bewertung des Schreibens bzw. der Texte, die die Lernenden verfasst haben. Der Text wird anhand der folgenden Kriterien bewertet: Formale Richtigkeit Gedanken formulieren - Aufgabenbewältigung. Die Aufgabe 6 bewertet die Fertigkeit der Lernenden, das Geschriebene zu bewerten und Fehler zu entdecken. Durch diese Aufgabe können die Lernenden das Geschriebene reflektieren. (S. Anhang 6)

\section{Analyse der Ergebnisse}

Die Analyse der Ergebnisse von den zwei Lerngruppen basiert zunächst auf statistischen Auswertungen und dann auf meinen Beobachtungen im Unterricht. 


\subsection{Quantitative statistische Auswertung der Ergebnisse}

Die Ergebnisse werden durch "SPSS" untersucht. Wegen der wenigen Zahl jeder Testgruppe werden die Ergebnisse von dem Verfahren "MannWhitney Test" analysiert.

\subsubsection{Die Überprüfung der ersten Hypothese}

Die erste Hypothese dieser Untersuchung lautet: Es sind abweichende Ergebnisse zwischen den Mittelwerten bzw. den Rängen der ersten Testgruppe (Mindmap) im Vor- und Nachtest bezüglich der Schreibkompetenz zugunsten des Nachtests zu erwarten.

\subsubsection{Die Überprüfung der zweiten Hypothese}

Die zweite Hypothese dieser Untersuchung lautet: Es sind abweichende Ergebnisse zwischen den Mittelwerten bzw. den Rängen der zweiten Testgruppe (kooperatives Schreiben) im Vor- und Nachtest bezüglich der Schreibkompetenz zugunsten des Nachtests zu erwarten.

\section{Tabelle (1)}

Differenzen zwischen den Mittelwerten der ersten Testgruppe (Mindmap) und der zweiten Testgruppe (Kooperatives Schreiben) im Nachtest bezüglich der Schreibkompetenz

\begin{tabular}{c|c|c|c|c|c|c|c}
\cline { 2 - 7 } & Zahl & Rang & $\begin{array}{c}\text { Summe } \\
\text { der } \\
\text { Ränge }\end{array}$ & $\begin{array}{c}\text { Wert } \\
\text { von } \\
\text { Z }\end{array}$ & $\mathrm{r}$ & $\mathrm{r}^{2}$ & $\mathrm{SN}$ \\
\hline $\begin{array}{c}\text { Testgruppe } \\
\text { "Mindmap" }\end{array}$ & 19 & 15.76 & 299.50 & 3.827 & 0.62 & $38 \%$ & 0.05 \\
\hline $\begin{array}{c}\text { Testgruppe } \\
\text { "Kooperatives } \\
\text { Schreiben" }\end{array}$ & 19 & 23.24 & 441.50 & 3.728 & 0.60 & $36 \%$ & 0.05 \\
\hline
\end{tabular}

Z: Unterschiede zwischen Rängen

r: Effizienz des Verfahrens

r²: Wirkungsgrad / Effect size 
SN: Signifikanz - Niveau / Signifikant level

Die Tabelle (1) zeigt, dass es Differenzen zwischen den Mittelwerten der beiden Testgruppen (Mindmap und Kooperatives Schreiben) im Vor- und Nachtest zugunsten des Nachtests bei einem Signifikanz-Niveau von 0.05 gibt. Der Wert $(Z)$ für die erste Testgruppe ist 3.827 und für die zweite Testgruppe ist 3.728. Die beiden Testgruppen verzeichnen Lernfortschritt aufgrund beiden Schreibverfahren.

\subsubsection{Die Überprüfung der dritten Hypothese}

Die dritte Hypothese der Untersuchung lautet: Es sind abweichende Ergebnisse zwischen den Mittelwerten bzw. Rängen der ersten Testgruppe (Mindmap) und der zweiten Testgruppe (Kooperatives Schreiben) im Vorund Nachtest bezüglich der Schreibphasen (Planen - Vorbereiten Organisieren - Strukturieren - Überdenken) zugunsten des Nachtests zu erwarten.

\section{Tabelle (2)}

Differenzen zwischen den Mittelwerten der ersten Testgruppe (Kooperatives Schreiben) und der zweiten Testgruppe (Mindmap)

bezüglich der fünf Schreibphasen

\begin{tabular}{c|c|c|c|c|c|c}
\cline { 2 - 6 } & $\begin{array}{c}\text { 1. } \\
\text { Frage } \\
\text { (Plane } \\
\text { n) }\end{array}$ & $\begin{array}{c}\text { 2. Frage } \\
\text { (Vorbereit } \\
\text { en) }\end{array}$ & $\begin{array}{c}\text { 3. Frage } \\
\text { (Organisie } \\
\text { ren) }\end{array}$ & $\begin{array}{c}\text { 4. Frage } \\
\text { (Strukturie } \\
\text { ren) }\end{array}$ & $\begin{array}{c}\text { 5. Frage } \\
\text { (Überdenk } \\
\text { en) }\end{array}$ & $\begin{array}{c}\text { gesa } \\
\text { mt }\end{array}$ \\
\hline $\begin{array}{c}\text { Mittelw } \\
\text { ert der } \\
\text { Ränge } \\
\text { G1 }\end{array}$ & 17.92 & 17.50 & 15.53 & 18.89 & 17.45 & 15.76 \\
\hline \hline $\begin{array}{c}\text { Mittelw } \\
\text { ert der } \\
\text { Ränge } \\
\text { G 2 }\end{array}$ & 21.08 & 21.50 & 23.47 & 20.11 & 21.55 & 23.24 \\
\hline \hline
\end{tabular}




\begin{tabular}{|c|c|c|c|c|c|c|}
\hline & $\begin{array}{c}1 . \\
\text { Frage } \\
\text { (Plane } \\
\text { n) }\end{array}$ & $\begin{array}{c}\text { 2. Frage } \\
\text { (Vorbereit } \\
\text { en) }\end{array}$ & $\begin{array}{l}\text { 3. Frage } \\
\text { (Organisie } \\
\text { ren) }\end{array}$ & $\begin{array}{c}\text { 4. Frage } \\
\text { (Strukturie } \\
\text { ren) }\end{array}$ & $\begin{array}{c}\text { 5. Frage } \\
\text { (Überdenk } \\
\text { en) }\end{array}$ & $\begin{array}{c}\text { gesa } \\
\text { mt }\end{array}$ \\
\hline $\begin{array}{c}\text { Wert Z } \\
\text { der } \\
\text { zwei } \\
\text { Gruppe } \\
\text { n }\end{array}$ & 0.928 & 1.155 & 2.224 & 0.342 & 1.153 & 2.077 \\
\hline \multicolumn{7}{|c|}{ r: 0.34} \\
\hline
\end{tabular}

Die statistischen Ergebnisse führen dazu, dass die beiden Verfahren (Mindmap und Kooperatives Schreiben) eine Wirkung bei der Entwicklung von Schreibkompetenz in allen Schreibphasen haben, aber es gibt Unterschiede zwischen den Testgruppen.

\subsubsection{Die Unterschiede zwischen den Mittelwerten der zwei Gruppen}

Die Ergebnisse der Untersuchung bzgl. Mittelwert und Rang haben erzielt, dass der Mittelwert der zweiten Testgruppe (Kooperatives Schreiben) größer als der Mittelwert der ersten Testgruppe (Mindmap) ist. Das Verfahren Kooperatives Schreiben ist wirksamer bei der Entwicklung von Schreibkompetenz als das Verfahren Mindmap.

Tabelle (3)

Differenzen zwischen der zwei Testgruppen zur Entwicklung der

Schreibkompetenz

\begin{tabular}{c|c|c}
\cline { 2 - 3 } \multicolumn{1}{c|}{} & $\begin{array}{c}\text { Mittelwert } \\
\text { vor }\end{array}$ & $\begin{array}{c}\text { Mittelwert } \\
\text { nach }\end{array}$ \\
\hline $\begin{array}{c}\text { Testgruppe 1 } \\
\text { Mindmap }\end{array}$ & 8.0 & 16.21 \\
\hline \hline Testgruppe 2 & 10.79 & 19.63 \\
Kooperatives Schreiben & & \\
\hline \hline
\end{tabular}


Eine Auswertung der Ergebnisse dieser Tabelle lässt erkennen, dass der größere Mittelwert bei der zweiten Testgruppe (kooperatives Schreiben) liegt.

\subsection{Qualitative Analyse der Ergebnisse}

Neben der quantitativen Analyse ist es wichtig, eine qualitative Analyse anzustellen. Nach der Umsetzung bzw. der Praxis kam ich zu den folgenden Ergebnissen, die sich aus Beobachtungen und aus bestimmten Tatsachen ergeben.

Die Lernenden finden die Lernmodule interessant. Sie freuen sich darauf, dass sie ihr Schreiben entwickeln können bzw. sich mit Schreiben beschäfitigen können. Sie sind der Meinung, dass das Schreiben als produktive sprachliche Fertigkeit schwierig ist und mehr Übung braucht. Sie äußern auch die Meinung, dass sie mehr an Kursen und ähnlichen Modulen teilnehmen möchten.

Die erste Testgruppe "Mindmap" erkannte den Unterschied zwischen "Wortigel " und "Mindmap". Zuvor hatten sie beide Begriffe verwechselt. Die Lernenden geben gute Beispiele von Mindmaps. Sie äußern die Meinung, dass die Schritte des Verfahrens Mindmap ihnen bei der Organisation von Gedanken helfen. Sie haben auch gelernt, Gedanken zuerst vor dem Schreiben zu formulieren und durch diese Gedanken Texte zu erstellen. Durch diese Vorüberlegungen können sie besser schreiben.

Die zweite Testgruppe "Kooperatives Schreiben" findet das gemeinsame Schreiben sehr sinnvoll. Sie arbeiten in der Regel gut mit und beteiligen sich aktiv am Unterrichtsgeschehen. Die Lernenden haben miteinander gelernt. Der Austausch von Informationen oder Erfahrungen hilft beim guten Schreiben. Auch gefällt ihnen der Schritt "Schreibkonferenz". Sie 
können differenziert, problemorientiert und Text nah argumentieren. Auch ist jeder bereit, das eigene Geschriebene (zuerst) und (dann) das Geschriebene von Anderen zu bewerten.

Einige Lernende sind leistungsschwach und schweigsam. Sie verhalten sich passiv und nehmen selten am Unterrichtsgeschehen teil.

Manche Lernende zeichnen sich jedoch durch relativ viele Fehler bei der Satzkonstruktion und der richtigen Anwendung grammatischer Kategorien (Tempus, Modus, Kasus) aus.

Das Resultat der Arbeit ist, dass die beiden vorgeschlagenen Verfahren eine Wirkung auf die Verbesserung von Schreibkompetenz haben. Die Lernenden verlangen mehr Übung und Anwendung. Die Fertigkeit Schreiben verlangt mehr Zeit als die anderen sprachlichen Fertigkeiten. Die Lernmodule motivieren die Lernenden, über bestimmte Themen zu schreiben und unterschiedliche Textsorten auszuprobieren.

\subsection{Schlussfolgerungen und Konsequenzen für DaF- Unterricht}

- Untersuchung von Mindmap bei anderen sprachlichen Kompetenzen konnte positive Ergebnisse zur Textproduktion haben.

- Die Umsetzung des kooperativen Schreibens beim Lernen und beim Erstellen von Texten ist effektiv und heutzutage im Unterricht gefragt.

- Das Schreibenlernen mit Hilfe von unterschiedlichen Verfahren und Strategien gilt als ein Weg zur Förderung von Kreativität und Fantasie der Lernenden.

- Die Förderung von Selbstbewertung und Fremdbewertung beim Schreiben konnte Lernfortschritt verursachen. 
- Das Unterrichtsklima verändert sich positiv bei der Verwendung von den neuen Schreibverfahren.

- Das gemeinsame Lernen steht im Vordergrund, und mehr Forschungen sollten im Bereich $\mathrm{DaF}$ die Effektivität von Kooperation und gemeinschaftlichem Lernen untersuchen.

- Die Fremdbewertung sollte bei verschiedenen sprachlichen Fertigkeiten untersucht werden.

\section{Literaturverzeichnis}

Benó, Eszter 2011: Kreatives Schreiben im DaF-Unterricht. Schreiben zu und nach literarischen Texten In: Neue Didaktik 1/2011. www.pedocs.de.pdf..2.08.2014.

Böttcher, Ingrid 2013: Anregungen zum kreativen Schreiben für den Schulunterricht, Ein Jugendwettbewerb des Meerbuscher Kluturskreises u. Des Rhein- Kreises Neuss, www.meerbuscher,kulturkreis.e.v.5.11.2013.

Bürkl. Anni / Edelmann, Gitta / Leister, Iris / Schwohl, Anette 2013: Kreatives Schreiben, von leeren Blatt zum fertigen Text .F.A Brockhaus / wissenmedia in der inmediaOne, GmbH, Gptersloh, München.

Canakkale, Niham Demiryay 2012: Kreativ-imitatives Schreiben im DaF-Unterricht: In, GFL, German as a foreign Language Jorunal, No.2$3 / 2012$.

Ciepielewska- Kaczmarek, Luiza 2011: Schreiben im frühen DaFUnterricht- Eine zu unrecht vernachlässigte Fähigkeit? In: Studie Linguisticie Eilolgici Online 9 Dipartimento di Linguistice Universita di Pisa. www.humnet.unipi.it.slifo/volg.2/2.8.2014.

Dinsel, Sabine / Reimann, Monika 2000: Fit für Zertifikat Deutsch B1 (Tipps und Übungen), Hueber Verlag, Ismaning, Deutschland. 
Frey, Evelyn 2007: Fit für Zertifikat Deutsch B2 (Tipps und Übungen), Hueber Verlag, Ismaning, Deutschland.

Gavrilas, Erwin 2012: kooperatives Schreiben im Deutschunterricht, Förderung der Schreibkompetenz am Beispiel von J.W. Goethe "Die Leiden des jungen Werthers", Murburg.

Heuer, Wiebke 2002: Mind Maps im Fremdsprachenunterricht, Max Hueber Verlag, Ismaning.

Heuer, Wiebke 2003: Arbeitsbuch "Arbeiten mit Mind Maps und Stichwörtern, Max Hueber Verlag, Ismaning.

Hinrichs, Beatrix 2011: Kreatives Schreiben, ein Weg zur Förderung der Schreibkompetenz von Schülern mit Deutsch als Zweitsprache im Deutschunterricht, Universität Duisburg Essen. www.kreatives$\underline{\text { schreibens,pro,daz,Stiftung,pdf.5.11.2013 }}$

Kaishauri, Sileikaite 2014: Zusatzmaterialien zum Beitrag "Texte Schreiben wie Häuser bauen im DaF-Unterricht: Strategien, Textaussteine und sprachliche Mittel als Argumentierens" Miteinander Heft 47. www.vdl.lt/sites/default(administratiorius/Downloads-Miteinander-

Schreiben-Diana.2013.11.03pdf.2.8.2014.

Kruse, Otto 2010: Lesen und Schreiben, der wichtige Umgang mit Texten im Studium, UVK Verlagsgesellschaft mbH, verlag Huter \& Roth KG, Wien.

Neuhaus, Gregor 2001: Forderung der Schreibkompetenz In: Busse, August / Neuhaus, Gregor / Steffens, Rudolf 2001: Schreibstrategien und Schreibprozesse, Förderung der Schreibkompetenz, Materialien für Unterricht und Lehrerbildung, Herausgegeben von Landesinstitut für Schule und Weiterbildung, Nordrhein Westfallen, Bönen. 
Neumann, Günther 2012: Mindmapping eine effektive Unterrichtsmethode. www.isb.bayern.de/download/pdf.6.08.2014.

Reich, K. (H.g) 2003: Methodenpool, Mind - Mapping, In: URL: http://methodenpool.uni-koeln.de.2003ff.5.11.2013.

Reinhold, Beate 2011: Prozessvalidierung von Schreibtests, Eine explorative Studie: In Deutsch als Fremdsprache, Zeitschrift zur Theorie und Praxis des Deutschunterrichts für Ausländer 2011, Heft 3. 48 Jahrgang, Universität Leipzig.

Schwarzbauer, Heike / Foltz, Holger / Macke, Wolfgang u,a. 2010: Schreibkompetenz fördern: Texte gestalten, überarbeiten, erstellen, Handreichungen und Materialien für den Deutschunterricht an beruflichen Schulen, Institut der deutschen Wirtschaft Köln, Kölner Universitätsverlag $\mathrm{GmbH}, \mathrm{Köln}$.

Steffens, Rudolf 2001: Förderung von Schreibprozessen durch Schreibberatung In: Busse, August / Neuhaus, Gregor / Steffens, Rudolf 2001: Schreibstrategien und Schreibprozesse, Förderung der Schreibkompetenz, Materialien für Unterricht und Lehrerbildung, Herausgegeben von Landesinstitut für Schule und Weiterbildung, Nordrhein Westfallen, Bönen.

Sturm, Afra 2008: kooperatives Schreiben- eine grundlegende Fähigkeit In: Rundschreiben Zentrum Lesen, Pädagogische Hochschule der FHNWInstitut Forschung und Entwicklung 15/2008.

Thiel, Bertram 2014: Dialogorientierte Mind-Mapping, Mind-Maps im Fachunterricht und ihre Umsetzung in Word for Windows, Microsoft Excel und Powerpoint, www.dialoisches.lernen.de/ www.arbeitsmethodik-fuerlehrende/ wwwarbeitsmethodik-im-unterricht.de.6.08.2014. 
Trim, John/ North, Brain/ Coste, Daniel (2001): Gemeinsamer europäischer Referenzrahmen für Sprachen: Lernen, Lehren, beurteilen, übersetzt von Quetz, Jürgen, Europarat, Straßburg.

Wolfrum, Jutta 2010: Kreativ schreiben, Gezielte Schreibförderung für jugendliche und erwachsene Deutschlernende (DaF/ DaZ), Hueber Verlag, München.

\section{Die Webseiten:}

www.bog.admin.chttranspk.15.01.2014,S,1.

www.post.ch/direkt/point20.01.2014,S,2. 


\section{أثر كلا من الخرائط الذهنية والكتابة الجماعية ــ دراسة مقارنةــ لتنمية كفاعة الكتابة لاى العي المتعلمين بقسم اللغة الألمانية بكلية التربية}

تعد الكتابة من المهارات الإنتاجية التى يكتسبها المتعلم أثناء تعلم اللغة الأجنبية والتى تتطلب المزيد من التطبيق والممارسة. و لأهمية الكتابة أثناء تعلم اللغة الألمانية كلغة أجنبية يهذف البحث

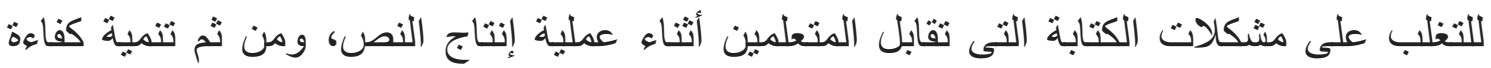
الكتابة لاى متعلمى قسم اللغة الألمانية بكلية التزبية. وللتغلب على بعض مشكلات الكتابة التى تم تحديدها اختارت الباحثة كلا من الخرائط الذهنية والكتابة الجماعية لتحديد تأثير هما على تتمية لتئه كفاءة الكتابة و المقارنة بينهما كاستير اتيجيات كتابة يمكن التغلب على مشكلات الكتابة المحددة من لهن خلالهما. ولقد صممت الباحثة مديول قائم على الخرائط الذهنية ، ومديول قائم على الكتابة الجماعية، يركز كل مديول على عمليات الكتابة التالية: التخطيط ـ الإعداد ـ التنظيم - الصياغة المراجعة. كما اشتمل كل مديول على تناول ثلاثة أنواع من النصوص لمعارسة الكتابة: المقالات و الخطابات الرسمية و الخطابات النخصية. وقد صممت الباحثة اختبار الكتابة لتقييم كفاءة الكتابة من خلال عمليات الكتابة السابق ذكرها. يعد الاختبار اختبارا قبليا وبعديا وتم تقسيم الطلاب إلى مجموعتين تجريبيتين. أوضحت نتئه نتيجة الاختبار القبلي أنه لا يوجد فرق بين مستوى المجموعتين التجريبيتين قبل تطبيق الدديولين. ثم ونمين

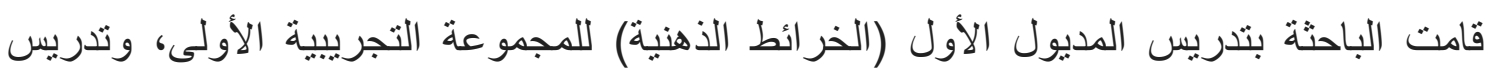

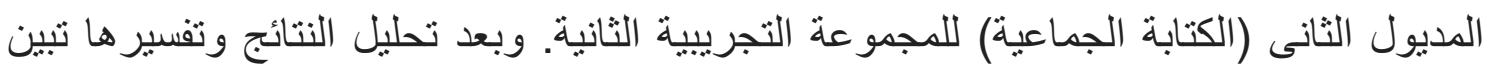
تأثير الاستراتيجيتين إيجابيا على كفاءة الكتابة، وبمقارنة نتائجهما تبين أن للكتابة الجماعية التأثير الأكبر عن الخر ائط الذهنية فى تتمية كفاءة الكتابة. و انتهى البحث بتئ بتقديم التحليل الكيفى للنتائج.

الكلمات المفتاحية : الخر ائط الذهنية ـ الكتابة الجماعية ـ إنتاج النص ـ كفاءة الكتابة 